\title{
The Ketogenic Diet and Its Effect on Bone Mineral Density: A Retrospective Observational Cohort Study
} \author{
Michèl A. Willemsen ${ }^{4}$ \\ 1 Department of Pediatrics, Amalia Children's Hospital, Radboud \\ Institute for Health Sciences, Radboud University Medical Center, \\ Nijmegen, The Netherlands \\ 2 Department of Radiology and Nuclear Medicine, Radboud University \\ Medical Center, Nijmegen, The Netherlands \\ ${ }^{3}$ Department of Gastroenterology and Dietetics, Radboud University \\ Medical Center, Nijmegen, The Netherlands \\ ${ }^{4}$ Department of Pediatric Neurology, Amalia Children's Hospital, \\ Radboud Institute for Health Sciences, Radboud University Medical \\ Center, Nijmegen, The Netherlands
}

Jos M.T. Draaisma ${ }^{1}$ Brieke M. Hampsink ${ }^{1}$ Marcel Janssen ${ }^{2} \quad$ Nicole B.M. van Houdt ${ }^{3}$

Neuropediatrics 2019;50:353-358
Address for correspondence Jos MT Draaisma, MD, PhD, Department of Pediatrics, Radboudumc Amalia Children's Hospital, PO Box 9101, 6500 HB Nijmegen, The Netherlands

(e-mail: jos.draaisma@radboudumc.nl).
Abstract
Keywords
- ketogenic diet tehrapy
- bone mineral density
- biphophonate therapy
- epilepsy

Background During long-term follow-up of children treated with the ketogenic diet therapy (KDT) have an increased incidence of bone fractures. However, the exact contribution of KDT to a decreased bone mineral density (BMD) remains unclear.

Objective This study aimed to evaluate (changes in) BMD in children treated with KDT and to evaluate whether intravenous bisphosphonate therapy may be effective.

Design In this retrospective, observational cohort study, all children treated with KDT from 2010 until 2018 at the Radboudumc Amalia Children's hospital were included. Patients who were on KDT for more than 6 months and who had at least two dualenergy X-ray (DXA)-scans were eligible for inclusion for longitudinal analysis. Z-scores of DXA-scans were compared over the course of time.

Results In 34 out of 68 patients, one or more lumbar DXA-scans were performed, with a mean lumbar $Z$-score of $-1.32 \pm 1.74$. Of these 68 patients, $8.8 \%$ got a fracture during KDT, and also $8.8 \%$ got kidney stones. In 20 patients, more than one DXA-scan was performed. A statistically not significant decrease in BMD (0.22 Z-score/year) was found. However, there was an increase in BMD in the five patients treated with intravenous bisphosphonate therapy. This was statistically significant in comparison to the nonbisphosphonate treated group $(p=0.034)$.

Conclusion Children on KDT have low normal BMD which may decrease further during KDT. For this reason monitoring of BMD is crucial, as is monitoring of kidney stones and hypercalciuria. Intravenous bisphosphonate therapy may have a positive effect, when other therapies have failed.
(C) 2019 Georg Thieme Verlag KG Stuttgart · New York
DOI https://doi.org/ 10.1055/s-0039-1693059. ISSN 0174-304X. 


\section{Introduction}

The ketogenic diet treatment (KDT) is a well-established, nonpharmacologic intervention for intractable childhood epilepsy. ${ }^{1,2}$ It was introduced in the 1920 s but its use decreased when the first antiepileptic drugs (AED) entered the market. ${ }^{2}$ However, over the past 20 years, an increase in its use can be seen. ${ }^{2}$ The diet has a high fat, adequate-protein (at least $1 \mathrm{gram} / \mathrm{kg}$ body weight), and low-carbohydrate composition that causes metabolic changes that mimic a (controlled) state of fasting., ${ }^{2,3}$ Often initiated with a brief fasting period and an in-hospital stay, changes in plasma ketones, insulin, glucose, glucagon, and free fatty acids occur within hours of starting the diet. ${ }^{4}$ In intractable childhood epilepsy, KDT is able to achieve a beneficial effect on seizure control, regardless of age or seizure type. ${ }^{3,5}$ As of yet, the exact mechanism of action is not completely understood and is thought to be multifactorial. ${ }^{4}$

Four major forms of KDT can be distinguished: the classic KDT, the modified Atkins diet (MAD), the medium chain triglyceride diet (MCT), and the low glycemic index diet (LGID). Their differences lie in the lipids to protein/carbohydrates ratio. ${ }^{3}$ Additional indications for KDT are, besides intractable childhood epilepsy, Glucose-1-transporter-deficiency-syndrome (GLUT1), pyruvate-dehydrogenase-deficiency (PDHD), and nonketotichyperglycinemia (NKH). Especially in GLUT1 and PDHD, KDT is recommended as the first choice treatment based on underlying pathophysiology. 6,7

During long-term follow-up of children treated with KDT, an increased incidence of bone fractures has been found, namely, up to $21 \%$ of the children during a follow-up period of 6 years or more. ${ }^{8}$ Bone fractures and decreased bone mineral density (BMD) are of concern for children maintained on KDT and chronic AED..$^{8-11}$ The effects of KDT, creating a high "acid load" via the ketone bodies, alterations in vitamin D levels, and lowering of growth factors, and the use of antiepileptic drugs, as well as wheel-chair dependence in a large proportion of patients with intractable epilepsies, all contribute to an increased risk for BMD loss. ${ }^{10,11}$ Nonetheless, the exact contribution of KDT to a decrease in BMD remains unclear. Prophylactic supplementation with calcium and vitamin D is mandatory for all children on the diet but sometimes seems to be failing to prevent continued BMD loss. ${ }^{10-12}$ However, during long-term follow-up of KDT, an increased incidence of kidney stones have also been found, mostly due to hypercalciuria. ${ }^{13,14}$ This makes prophylactic supplementation of calcium and vitamin $\mathrm{D}$ challenging.

Screening with dual-energy X-ray (DXA) scans to evaluate for a diminished BMD is recommended in children on KDT for over 2 years by 12 of the 25 (48\%) centers participating in the International Ketogenic Diet Study Group. ${ }^{3}$ The main recommendation of the recent report of this study group was that this should be standard and abnormal results should lead to possible interventions and scans should be repeated a year afterwards. Despite this recent endorsement, there is no well-defined role for the use of bone scanning and treatments (in this setting) to reduce the risk of BMD loss and fractures. ${ }^{3}$
This study aims to evaluate changes in BMD measured with DXA-scans in children treated with KDT, and to evaluate the possible use of intravenous bisphosphonates to treat osteoporosis and decreased BMD, especially in patients with kidney stones. ${ }^{13,15}$

\section{Methods}

\section{Study Design}

All children of ages ranging from 0 to 18 years who underwent KDT at the Radboudumc Amalia Children's hospital from January 1, 2010 until August 1, 2018 were included. Longitudinal analyses of the BMD status were performed on the subgroup of children who were on KDT for at least 6 months and had at least two DXA-scans. The DXA-scanner used was a Hologic Discovery A S/N 85606 densitometer, with a scanning time of 10 seconds and an exposition of $0.04 \mathrm{mGy}$ per scan. The coefficient of variation was $<1 \%$. Areal BMD, bone mineral content (BMC) and soft tissue composition were assessed with scans taken at the hip (hip) and lumbar spine (lumbar). Because accurate measurement of height was often not possible due to contractures and scoliosis, $Z$-scores were calculated using age- and sex-specific normative data provided by Hologic. Only the $Z$-scores from the lumbar spine were included for analysis, as these are more reliable. ${ }^{16}$ These data were supplemented with the indication for KDT, calcium, and vitamin D supplementation, AED therapy during KDT, wheel-chair dependence, experienced fractures during KDT, intravenous bisphosphonate therapy, ultrasounds of kidneys, and urinary calcium to creatinine ratio ( $\mathrm{Ca}: \mathrm{Cr}$ ). ${ }^{17,18}$ on the use of other relevant drugs, like potassium citrate and hydrochloorthiazide, which are prescribed to prevent renal complications of KDT, were also collected. ${ }^{14}$

\section{Dietary Treatments}

Children, in this study, were prescribed a KDT in various ratios, namely, a classic KDT, MCT diet, MAD, or LGID. Caloric intake, protein content and, thus, ketogenic dietary ratio were routinely adjusted throughout the treatment period if necessary, for example, to respond to weight gain or loss, and, obviously, the effects on seizure frequency. An intention-to-treat analysis was applied to patients who changed KDT subtype during treatment; henceforth, their starting diet was leading. According to the Dutch national guideline, all children were supplemented with multivitamins containing minerals at a vitamin concentration, approximately recommended for their age, and additional calcium and vitamin D when appropriate. ${ }^{3,19}$

\section{Statistical Analyses}

Statistical analyses were conducted using IBM SPSS Statistics, 25.0 for Windows (SPSS, IBM Corp., Armonk, New York, United States). Descriptive statistics were used for to determine the patients' eligible for inclusion in the longitudinal analysis of the BMD status. For the longitudinal analysis of the BMD status, lumbar spine $Z$-scores of the first DXA-scan were compared with lumbar $Z$-scores of the last DXA-scan, with an additional correction for time on KDT (in subanalyses only 
DXA-scans performed after 6 months on KDT were included). Wilcoxon's signed rank tests were used to calculate this difference in $Z$-score over the course of time. Thereafter, a coefficient of the lumbar DXA-scan results was calculated using a mixed logistic regression model. The means of this coefficient were subsequently compared in-between groups using Mann-Whitney $U$-tests. In all analyses, two-tailed $p<0.05$ was considered statistically significant.

This article does not contain any studies with human participants performed by any of the authors. For this type of study, formal consent is not required. According to the Medical Ethics Committee (CMO) of the district Arnhem/ Nijmegen, no ethical approval was required for this study.

\section{Results}

In total, 68 children were treated with KDT. The mean age at start of the KDT was $5.7 \pm 4.3$ years and the mean time on KDT was $25.6 \pm 24.8$ months (-Table 1). In 34 patients, one or more lumbar DXA-scans had been performed, with a mean lumbar $Z$-score of $-1.32 \pm 1.74$ on the initial DXA-scan. Of the 68 patients, $6(8.8 \%)$ patients got one or more long-bone fractures during KDT, of which 2 were acquired after a mild trauma, and the causes of the other fractures were unknown/ nontraumatic.

Out of the group of 68 patients, 35 patients (52\%) underwent at least one renal ultrasound at any point during treatment. Six out of 68 patients (8.8\%) developed kidney

Table 1 Characteristics of 68 patients treated with KDT

\begin{tabular}{|c|c|}
\hline Male & $36(52.9 \%)$ \\
\hline Age at initiation of KDT (y) & $5.7 \pm 4.3$ \\
\hline Mean time on KDT (mo) & $25.6 \pm 24.8$ \\
\hline Fractures during KDT & $6(8.8 \%)$ \\
\hline \multicolumn{2}{|l|}{ Indication of KDT } \\
\hline - Epilepsy (not GLUT1DS and not PDHC) & $54(79.5 \%)$ \\
\hline - GLUT1DS & $9(13.2 \%)$ \\
\hline - PDHD & $2(2.9 \%)$ \\
\hline - Other & $3(4.4 \%)$ \\
\hline \multicolumn{2}{|l|}{ KDT subtype: } \\
\hline - Classic KDT & $46(67.6 \%)$ \\
\hline - MAD & $13(19.1 \%)$ \\
\hline - $\mathrm{MCT}$ & $2(2.9 \%)$ \\
\hline - LGID & $5(7.4 \%)$ \\
\hline - Other & $1(1.5 \%)$ \\
\hline AED during KDT & $61(89.7 \%)$ \\
\hline \multicolumn{2}{|l|}{ Supplemental dietary intake } \\
\hline - Calcium & $24(35.3 \%)$ \\
\hline - Vitamin D & $39(57.4 \%)$ \\
\hline
\end{tabular}

Abbreviations: AED, anti-epileptic drugs; GLUT1DS, Glucose-1-transporter deficiency syndrome; KDT, ketogenic diet therapy; LGID, low glycemic index diet; MAD, modified Atkins diet; $M C T$, medium chain triglyceride; PDHD, Pyruvate dehydrogenase deficiency. stones during treatment. The first mean urinary $\mathrm{Ca}: \mathrm{Cr}$ ratio in the 16 patients in whom it was measured was elevated $(1.02 \pm 0.93 \mathrm{mmol} / \mathrm{mmol})$, and although therapeutic interventions were taken, the last measured $\mathrm{Ca}: \mathrm{Cr}$ ratio in five of these patients was still not normalized $(0.84 \pm 0.81$ $\mathrm{mmol} / \mathrm{mmol}[n=5]$ ) (reference values: $0.04-0.7 \mathrm{mmol} /$ mmol). ${ }^{18}$ AED were used in $89.9 \%$ of the patients during KDT, whereby zonisamide was used in 27 out of 68 patients (39.7\%). In 10 (14.7\%) patients, potassium citrate and/or hydrochloorthiazide were used. We noticed a trend of prescribing potassium citrate immediately at initiation of the diet in patients who started KDT more recently.

Out of the 68 enrolled children a total of 20 patients met inclusion criteria for longitudinal BMD status analysis. Their clinical characteristics are presented in $\mathbf{- T a b l e ~} \mathbf{2}$. The mean age at initiation of KDT was 6.61 years ( $0.83-15.48$ years). Of these patients, four (20\%) experienced one or more fractures at any time during KDT. Thirteen (65\%) patients were wheelchair dependent. Mean DXA-scan results are presented in - Table 3. The first DXA-scan was made $14.8 \pm 21.0$ months after start of KDT. Patients had two to five DXA scans. We compared the mean lumbar DXA-scan $Z$-scores of the first scan with the last scan. Overall there was a trend toward decrease in BMD, but this was not statistically significant $(p=0.760)$. To correct for the large time differences and to increase the reliability of a possible correlation between KDT and the BMD, we made exploring corrections. After only including scans performed after 6 months on KDT

Table 2 Characteristics of the 20 patients included for longitudinal BMD status analysis

\begin{tabular}{|c|c|}
\hline Male & $11(55 \%)$ \\
\hline Age at initiation (y) & $6.6 \pm 3.7$ \\
\hline Mean time on KDT (mo) & $39.55 \pm 23.12$ \\
\hline Fractures during KDT & $4(20 \%)$ \\
\hline \multicolumn{2}{|l|}{ Indication of KDT } \\
\hline - Epilepsy & 15 (75\%) \\
\hline - GLUT1DS & $3(15 \%)$ \\
\hline - $\mathrm{PDHD}$ & $2(10 \%)$ \\
\hline \multicolumn{2}{|l|}{ KDT subtype: } \\
\hline - Classic KDT & $15(75 \%)$ \\
\hline - MAD & $3(15 \%)$ \\
\hline - $\mathrm{MCT}$ & $1(5 \%)$ \\
\hline - LGIT & $1(5 \%)$ \\
\hline AED during KDT & $18(90 \%)$ \\
\hline \multicolumn{2}{|c|}{ Supplemental dietary intake } \\
\hline - Calcium & $16(80 \%)$ \\
\hline - Vitamin D & $16(80 \%)$ \\
\hline
\end{tabular}

Abbreviations: AED, anti-epileptic drugs; GLUT1DS, Glucose-1-transporter deficiency syndrome; KDT, ketogenic diet therapy; LGID, low glycemic index diet; MAD, modified Atkins diet; MCT, medium chain triglyceride; PDHD, Pyruvate dehydrogenase deficiency. 
Table 3 Wilcoxon's signed rank test of lumbar DXA-scan results

\begin{tabular}{|l|l|l|l|l|l|}
\hline Analysis & $\begin{array}{l}\text { First lumbar } \\
\text { Z-score }\end{array}$ & $\begin{array}{l}\text { Last lumbar } \\
\text { Z-score }\end{array}$ & $\begin{array}{l}\text { Difference in } \\
\text { Z-score }\end{array}$ & $\begin{array}{l}\text { Time interval in } \\
\text { months between } \\
\text { first and last } \\
\text { lumbar Z-score }\end{array}$ & $p$-Value \\
\hline All patients $(n=20)$ & $-1.89 \pm 1.65$ & $-2.01 \pm 2.15$ & $-0.11 \pm 1.26$ & $22.1 \pm 9.8$ & 0.76 \\
\hline $\begin{array}{l}\text { Only those who had their first } \\
\text { DXA after 6 months on KDT }(n=15)\end{array}$ & $-1.70 \pm 1.45$ & $-1.84 \pm 1.88$ & $-0.14 \pm 1.29$ & $20.2 \pm 8.7$ & 0.70 \\
\hline $\begin{array}{l}\text { Only patients without biphoshonate } \\
\text { therapy }(n=15)\end{array}$ & $-1.80 \pm 1.82$ & $-2.19 \pm 2.39$ & $-0.39 \pm 1.37$ & $21.3 \pm 10.1$ & 0.22 \\
\hline $\begin{array}{l}\text { Only patients with biphosphonate } \\
\text { therapy }(n=5)\end{array}$ & $-2.16 \pm 1.39$ & $-1.50 \pm 1.36$ & $0.66 \pm 0.59$ & $24.4 \pm 9.4$ & 0.08 \\
\hline
\end{tabular}

Abbreviations: DXA, dual-energy X-ray; KDT, ketogenic diet therapy.

$(p=0.701)$ and excluding patients treated with intravenous bisphosphonates $(p=0.221)$ we did not find a significant decrease. Although in all these analysis there is a decline in lumbar $Z$-score, this never reached a statistically significance. The mean decrease in lumbar DXA-scan was $0.22 \mathrm{Z}$-score/year in patients without intravenous bisphosphonate therapy.

The same evaluation was made for patients treated with intravenous bisphosphonate therapy to evaluate the effectiveness of this fracture-preventive treatment. The mean BMD increased, though again not statistically significant $(p=0.080)$. Correction for the timing of the DXA-scans, comparing the first scan performed after at least 6 months on KDT with the last performed DXA-scan showed also no statistically significant results $(p=0.340)$.

However, when the BMD change of patients treated with intravenous bisphosphonate therapy was compared with patients without this treatment, a significant difference was found $(p=0.034)$. AED therapy during KDT did not statistically influence the BMD change $(p=0.21)$ nor did wheel-chair dependency $(p=0.96)$

So, a trend to a decline in lumbar BMD can be found in patients without intravenous bisphosphonate therapy opposed to an increase in lumbar BMD in patients treated with this therapy.

Of the children who got intravenous bisphosphonate therapy, one had multiple fractures and met the International Society for Clinical Densitometry (ISCD) criteria pediatric osteoporosis, ${ }^{20}$ one had one fracture and kidney stones, two had declining BMD (in one patient the lumbar BMD Z-score declined from -2.3 to -2.4 , in the other declined from -2.6 to -3.1 ) and kidney stones, and one had a declining lumbar BMD $Z$-score from -2.6 to -3.1 . In the patients with kidney stones, the symptoms and/or the kidney stones diminished or disappeared.

\section{Discussion}

This study describes the BMD status in a population of 68 children treated with KDT. On average, all patients included had a decreased mean lumbar spine $Z$-score of -1.32 . This is lower than the $Z$-scores of -0.93 and -0.99 in earlier studies on this topic. ${ }^{11,12}$ However, they measured the initial BMD before the start of the treatment. Our national guideline recommend to perform only DXA-scans when there are also other risk factors as wheel-chair dependency or the persistent use of AED's. ${ }^{19}$ Till 2017, we started to measure BMD at the age of 10 years. We changed this to the age of 4 years, which is the reason that most of our first DXA-scans were not performed at the start of KDT.

Thus far, there have been only abovementioned two studies examining the longitudinal progress of BMD of children on KDT. ${ }^{11,12}$ The first study was published in 2008 and described 25 children aged 5 years or more on KDT. KDT resulted in a significantly loss of BMD in 15 months' time, with $0.6 Z$-score/year. ${ }^{11}$ In the second study, published in $2017,{ }^{12} 29$ children on KDT for a minimum of 6 months were included. There was a trend toward a reduction in lumbar spine $Z$-score of $0.16 /$ year $(p=0.07)$. A trend toward a reduction in BMD (0.22 Z-score/year), while on KDT was also found in our study. There are two possible explanations for the difference between the results in the first study of Bergqvist et al and the results of the second study of Simm et al and our study. ${ }^{11,12}$ The first explanation might be that patients were more or less ambulant. In our study and the second study, less than $40 \%$ of patients were ambulant, and in the first study more than $80 \%$. Simm et al found that more mobile patients had a statistically significant more decline in the BMD $Z$-score. ${ }^{12}$ They speculated that maybe more ambulant subjects are more susceptible to metabolic derangements because of their higher bone turnover. The other possible explanation could be a difference in KDT. All patients in the first study used a 4:1 ketogenic diet, in the second study patients used a 2:1 to $4: 1$ ketogenic diet and in our study patients used a ketogenic diet in various ratios.

We are the first who report on the effect of intravenous bisphosphonate therapy during KDT and found a statistically significant difference between the first and last performed scan in patients with intravenous bisphosphonates treatment in comparison to patients without this treatment in our study group.

In the 2018 International Ketogenic Diet Study group consensus-based guidelines, the authors stated that it might 
be advantageous for children to have periodic DXA screening for assessment of bone health. ${ }^{3}$ Also, they recommend that if DXA results are obtained, abnormal results should be leading to possible interventions and scans should be repeated one year afterward. They recommend that both calcium and vitamin $\mathrm{D}$ should be provided at the recommended daily allowance, although about half of the consensus group provides additional vitamin $\mathrm{D}$ beyond the recommended dietary allowance (RDA) guidelines. However, it was recognized that one of the problems is that almost $40 \%$ of patients may have an elevated urine calcium/creatinine ratio at baseline (i.e., at start of KDT) that increased to $75 \%$ after 6 months on diet. ${ }^{13}$ The high-normal rate (8.8\%) of kidney stones in this study may be due to the high rate of calcium supplementation with an increased urine calcium/creatinine ratio, and the high rate of the use of zonisamide. For this reason we now measure vitamin $\mathrm{D}$ and the urine calcium/creatinine ratio routinely during follow-up. More recently we started with the use of oral citrates to prevent the formation of kidney stones. $^{13,14,17,21}$

There are no studies on the use of bisphosphonates in children with KDT. ${ }^{15}$ Treatment with intravenous biphosphonates should be considered in patients with genetic forms of osteoporosis or those whose risk factor cannot be mitigated or when there is a low likelihood of spontaneous recovery. ${ }^{22}$

The primary function of bisphosphonates is to inactivate osteoclasts which results in cortical and trabecular bone thickening. This makes bones wider, denser, and stronger. A common side effect is hypocalcaemia and hypophosphatemia which may help to treat current kidney stones. This is the reason why we ultimately also treated five patients with a bisphosphonate (intravenous pamidronate $1 \mathrm{mg} / \mathrm{kg}$ four times/year). They had a low, declining BMD with or without kidney stones. With intravenous bisphosphonate treatment the mean BMD increased, instead of decreased. This was statistically significant in comparison to the nonbisphosphonate group ( $p=0.034)$. Moreover, the kidney stones diminished or disappeared. Recently, Nasomyont et al published a study in which they also treated $19 \%$ of the patients with intravenous biphosphonate infusion out of the ISCD criteria. ${ }^{23}$ One of these indications was low BMD without a history of fractures, another low BMD in association with kidney stones.

Our study has its strengths and limitations. The main strength is the length of the follow-up. In the published studies on the effect of KDT on BMD the mean follow- up was 15 and 25 months, respectively, in contrast to the 39.5 months follow-up in this study. ${ }^{11,12}$ Moreover, this is the first study in which the use of intravenous biphophonate therapy is described in children on KDT.

The main limitation is that the group is not uniform in the application of DXA-scans . In only 34 (50\%) patients, a DEXAscan was performed, and in only 20 more than one DXA-scans were available. This was due to the fact that patients were not randomized and DXA-scans were, according to our hospitalprotocol, at first only performed in patients above the age of 10 years. No fixed time slots were used. However, this is to be expected due to the retrospective nature of this study. Furthermore, body weight of patients was not embraced and is found to influence BMD. ${ }^{11}$ Moreover, not all the patients had the same KDT, but the effect of the different KDT's on BMD could not be analyzed, due to the low number of patients.

This study might augment the findings of earlier literature that patients on the ketogenic diet undergo a deterioration of their bone mineral density, which may be related to the use of KDT. The fact that in our population, 6 out of 68 patients on KDT sustained fractures highlights the potential clinical relevance of these findings, given the morbidity that accompanies such events. Our findings once again highlight the need for close monitoring of bone health in individuals on KDT, including protocols for modifiable parameters, such as calcium intake, vitamin D deficiency and activity levels. Developing kidney stones remains a major complication of $\mathrm{KDT}$, and has to be taken into account when assessing a patient on the diet. Urine $\mathrm{Ca}: \mathrm{Cr}$ ratios and renal ultrasounds have to be performed during follow-up. ${ }^{3}$

\section{Conclusion}

This study shows that KDT might be associated with decreased bone mass accrual, and supports previously suggested findings that bone health monitoring is important in this high-risk group. For this reason, close monitoring of BMD is crucial, as is the monitoring of the development of kidney stones. Intravenous bisphosphonate therapy may have a positive effect on BMD and may reduce kidney stones, when other therapies have failed.

Conflict of Interest

None declared.

\section{References}

1 Keene DL. A systematic review of the use of the ketogenic diet in childhood epilepsy. Pediatr Neurol 2006;35(01):1-5

2 Wheless JW. History of the ketogenic diet. Epilepsia 2008;49 (Suppl 8):3-5

3 Kossoff EH, Zupec-Kania BA, Auvin S, et al; Charlie Foundation; Matthew's Friends; Practice Committee of the Child Neurology Society. Optimal clinical management of children receiving dietary therapies for epilepsy: updated recommendations of the International Ketogenic Diet Study Group. Epilepsia Open 2018; 3(02):175-192

4 Bough KJ, Rho JM. Anticonvulsant mechanisms of the ketogenic diet. Epilepsia 2007;48(01):43-58

5 Neal EG, Chaffe H, Schwartz RH, et al. The ketogenic diet for the treatment of childhood epilepsy: a randomised controlled trial. Lancet Neurol 2008;7(06):500-506

6 Klepper J, Leiendecker B. GLUT1 deficiency syndrome-2007 update. Dev Med Child Neurol 2007;49(09):707-716

7 Wexler ID, Hemalatha SG, McConnell J, et al. Outcome of pyruvate dehydrogenase deficiency treated with ketogenic diets. Studies in patients with identical mutations. Neurology 1997;49(06):1655-1661

8 Groesbeck DK, Bluml RM, Kossoff EH. Long-term use of the ketogenic diet in the treatment of epilepsy. Dev Med Child Neurol 2006;48(12):978-981

9 Kang HC, Chung DE, Kim DW, Kim HD. Early- and late-onset complications of the ketogenic diet for intractable epilepsy. Epilepsia 2004;45(09):1116-1123 
10 Bergqvist AG, Schall JI, Stallings VA. Vitamin D status in children with intractable epilepsy, and impact of the ketogenic diet. Epilepsia 2007;48(01):66-71

11 Bergqvist AG, Schall JI, Stallings VA, Zemel BS. Progressive bone mineral content loss in children with intractable epilepsy treated with the ketogenic diet. Am J Clin Nutr 2008;88(06):1678-1684

12 Simm PJ, Bicknell-Royle J, Lawrie J, et al. The effect of the ketogenic diet on the developing skeleton. Epilepsy Res 2017;136:62-66

13 Furth SL, Casey JC, Pyzik PL, et al. Risk factors for urolithiasis in children on the ketogenic diet. Pediatr Nephrol 2000;15(1,2):125-128

14 Sampath A, Kossoff EH, Furth SL, Pyzik PL, Vining EP. Kidney stones and the ketogenic diet: risk factors and prevention. J Child Neurol 2007;22(04):375-378

15 Saraff V, Högler W. Endocrinology and adolescence: osteoporosis in children: diagnosis and management. Eur J Endocrinol 2015; 173(06):R185-R197

16 Messina C, Lastella G, Sorce S, et al. Pediatric dual-energy X-ray absorptiometry in clinical practice: what the clinicians need to know. Eur J Radiol 2018;105:153-161

17 Kossoff EH, Pyzik PL, Furth SL, Hladky HD, Freeman JM, Vining EP. Kidney stones, carbonic anhydrase inhibitors, and the ketogenic diet. Epilepsia 2002;43(10):1168-1171
18 Matos V, van Melle G, Boulat O, Markert M, Bachmann C, Guignard JP. Urinary phosphate/creatinine, calcium/creatinine, and magnesium/creatinine ratios in a healthy pediatric population. J Pediatr 1997;131(02):252-257

19 Van der Louw EJTM, Van den Hurk TAM, Catsman-Berrevoets CE. Guideline for professionals of the ketogenic diet treatment for children with intractable epilepsy and metabolic diseases 2015 Available (in Dutch) at: https://www.dietistenvoorspierziekten. nl/upload/docs/zorgpad-ketogeen-dieet-behandeling-bij-kinderen-versie-mei-2015_0.pdf. Accessed June 17, 2019

20 Gordon CM, Leonard MB, Zemel BS; International Society for Clinical Densitometry. 2013 Pediatric Position Development Conference: executive summary and reflections. J Clin Densitom 2014;17(02):219-224

21 McNally MA, Pyzik PL, Rubenstein JE, Hamdy RF, Kossoff EH. Empiric use of potassium citrate reduces kidney-stone incidence with the ketogenic diet. Pediatrics 2009;124(02):e300-e304

22 Ward LM, Konji VN, Ma J. The management of osteoporosis in children. Osteoporos Int 2016;27(07):2147-2179

23 Nasomyont N, Hornung LN, Gordon CM, Wasserman H. Outcomes following intravenous bisphosphonate infusion in pediatric patients: a 7-year retrospective chart review. Bone 2019;121:60-67 